\title{
Significant improvement of bone mineral density and bone turnover markers by denosumab therapy in bisphosphonate-unresponsive patients
}

\author{
M. Kamimura ${ }^{1}$-Y. Nakamura ${ }^{2,3} \cdot$ S. Ikegami $^{2}$. \\ S. Uchiyama ${ }^{2} \cdot$ H. Kato ${ }^{2} \cdot$ A. Taguchi ${ }^{4}$
}

Received: 25 July 2016 / Accepted: 31 August 2016/Published online: 20 September 2016

(C) The Author(s) 2016. This article is published with open access at Springerlink.com

\begin{abstract}
Summary Bone mineral density (BMD) sometimes cannot be improved by long-term bisphosphonate (BP) therapy in osteoporosis (OP). This study showed that lumbar as well as hip BMD significantly increased after denosumab treatment in patients not responsive to BPs. Thus, denosumab may be a strong OP treatment option for BP-unresponsive patients.

Introduction BMD sometimes cannot be improved by longterm BP therapy.

Methods We administered denosumab to osteoporotic patients with a poor response to BPs who had been taking them for 2 years or longer. Ninety-eight women with BP-poor responsive $\mathrm{OP}$ were enrolled in this study. Mean (standard deviation [SD]) age was 71.2 (6.9) years and mean (SD) duration of BP treatment was 59.9 (34.3) months. We distinguished BP responders from non-responders based on changes in BMD values at denosumab commencement (baseline) from 2 years beforehand.

Results There were no significant differences in age, duration of BP use, bone turnover markers, or BMD at baseline between the groups. Prior to denosumab, BMD had increased
\end{abstract}

Y. Nakamura

yxn14@aol.jp

1 Center of Osteoporosis and Spinal Disorders, Kamimura Orthopedic Clinic, Kotobuki 595-17, Matsumoto 399-0021, Japan

2 Department of Orthopaedic Surgery, Shinshu University School of Medicine, Asahi 3-1-1, Matsumoto 390-8621, Japan

3 Department of Orthopedic Surgery, Showa-Inan General Hospital, Akaho 3230, Komagane 399-4117, Japan

4 Department of Oral and Maxillofacial Radiology, Matsumoto Dental University, 1780 Gobara Hirooka, Shiojiri 399-0781, Japan significantly in responders and decreased significantly in nonresponders. Bone turnover markers had decreased significantly at 4 months of denosumab treatment $(P<0.001)$ and lumbar and hip BMD were significantly increased at 1 year of therapy in both groups $(P<0.001)$. Simple correlation coefficients were -0.337 for lumbar and -0.339 for hip BMD changes (both $P=0.001$ ) before and after denosumab treatment. Both at the lumbar spine and hips, decreased BMD before denosumab therapy was significantly associated with an increase in BMD at 1 year of treatment (spine, $t$ value $=-3.502, P=0.001, R=0.113$; hip, $t$ value $=-3.526$, $P=0.001, R=0.115$ ).

Conclusions These results suggest that denosumab may be a strong OP treatment option for BP-unresponsive patients.

Keywords Bisphosphonate $\cdot$ Bisphosphonate-unresponsive $\cdot$ Bone turnover markers $\cdot$ Denosumab · Osteoporosis

\section{Introduction}

Osteoporosis (OP) is a widespread skeletal disorder requiring long-term care and management. The purpose of osteoporotic treatment is the prevention of fractures to maintain activities of daily living and thereby reduce mortality.

Current therapies for OP are based on our understanding of bone biology. Receptor activator of nuclear factor-kB ligand (RANKL) is a cytokine that is essential for osteoclast differentiation, activation, and survival. Denosumab, a fully human monoclonal antibody against RANKL, has been shown to selectively inhibit osteoclastogenesis. Consequently, the drug strongly abrogates bone resorption, increases bone mineral density (BMD), and prevents fragility fractures $[1,2]$. The very recent FREEDOM open-label extension study also demonstrated that nonvertebral fracture rate was decreased for up 
to 7 years after denosumab treatment and that BMD values increased linearly [3]. Thus, denosumab is considered as one of the best therapeutic options for osteoporotic patients with respect to increased BMD, improvement in bone turnover markers, and prevention of fractures.

Bisphosphonates (BPs) are the most common drugs for osteoporotic treatment. With their bone anti-resorptive properties, BPs improve bone turnover, and consequently, increase BMD and prevent fractures [4]. Nitrogen-containing BPs inhibit farnesyl pyrophosphate synthetase in the mevalonate pathway in osteoclasts [5] to suppress the function of osteoclasts and induce their apoptosis, thereby, inhibiting osteoclastic activity. The alendronate (ALN), risedronate (RIS), and minodronate (MIN) used in the present study are such nitrogen-containing BPs. BPs and denosumab share the same mechanism of osteoclastogenesis inhibition.

BPs increase BMD remarkably in postmenopausal patients with OP, especially over the first few years [6]. However, the effectiveness of BPs on BMD diminishes over longer treatment periods [7], and so, the continuous use of these drugs in osteoporotic patients is sometimes limited [8]. In such cases, alternative drugs, such as denosumab, are required, although little is known on the improvement bone turnover and/or $\mathrm{BMD}$ by denosumab in primary $\mathrm{OP}$ refractory to $\mathrm{BP}$ treatment.

At our institutions, BPs were principally used for osteoporotic patients with low BMD prior to the approval of denosumab or parathyroid hormone (PTH) in Japan. In our experience, $\mathrm{BMD}$ values increase to various degrees in most patients in the initial few years of BP treatment and satisfactorily increased BMD is frequent. Afterwards, however, some cases emerge in which BMD values plateau, or even decrease, in the long term, regardless of the BP used.

The reproducibility and measurement accuracy of BMD with dual-energy X-ray absorption (DXA) are high using a scanning table [9]. However, not only hip, but also lumbar, BMD values vary since obvious measurement errors occasionally occur in daily practice. Therefore, observational BMD values in each patient do not change in a linear fashion, and the judgment of treatment effectiveness based on a single $\mathrm{BMD}$ recording is not appropriate; minus measurement errors of DXA would be likely evaluated as non-responsive cases, the drugs would be changed, and a minus bias in which BMD values increase after the treatment alteration would occur. To address this issue, it is preferable to evaluate the therapy effectiveness based on multiple value changes over time.

Since its approval in Japan in 2013, we have routinely prescribed denosumab for cases of poor BP response with low BMD where increased BMD even decreased after longterm BP treatment. In this study, we first determined treatment responsiveness by measurement of lumbar and/or total hip BMD values at least once every 6 months for 2 years. To our knowledge, this is the first report on switching from long-term BP therapy to denosumab in postmenopausal primary OP refractory to BPs.

\section{Materials and methods}

\section{Subjects and measurements}

In the period from June 2013 to February 2015, we recruited 118 patients with low lumbar or hip BMD values undergoing long-term BP treatment, who were only primary OP after careful differential diagnosis. We defined poor BP responders as individuals in whom lumbar or hip BMD had not apparently increased with chronic BP administration. Ultimately, 98 women aged between 54 and 84 years participated in this study after providing informed consent. These subjects had received 5 BMD measurements at 6-month intervals during the 2 years of BP treatment preceding denosumab treatment. Twenty women had not completed all tests and were dropped from the study. One man was excluded to avoid gender bias. ALN, RIS, and MIN were adopted in various regimens as long-term BP pre-treatment. We did not examine the effects of individual BP drugs since they were routinely changed for patients exhibiting low responsiveness. In the patients treated with $\mathrm{BPs}$, vitamin D and Ca supplementation were not used in all of the cases. On the other hand, $400 \mathrm{IU}$ vitamin D and $610 \mathrm{mg} \mathrm{Ca}$ supplementation were used in all of the patients treated with denosumab. All treatments were substituted from BPs to denosumab at the baseline. Mean (standard deviation [SD]) age of the 98 subjects was $71.2(6.9)$ years and mean (SD) duration of BP treatment prior to denosumab was 59.9 (34.3) months.

BMD was measured using a DXA fan-beam bone densitometer (Lunar Prodigy; GE Healthcare Bio-Sciences Corp., Piscataway, NJ, USA) at the lumbar 1-4 level of the posteroanterior spine and at the bilateral hips as the mean of the right and left sides. Fracture sites were avoided for BMD evaluation. Following the change to denosumab, BMD values were quantified every 4 months for 1 year. Coefficients of variation of the BMD measurement at lumbar spine and hip were 0.6 and $0.5 \%$, respectively. Least significant changes of these measurement were accordingly 1.6 and $1.5 \%$, respectively [9].

At baseline and 4 months of denosumab treatment, we measured serum bone-specific alkaline phosphatase (BAP) as a marker for bone formation and urinary N-terminal telopeptide of type I collagen corrected by creatinine (NTx) as a bone resorption marker. Serum BAP was evaluated using a chemiluminescent enzyme immunoassay. Urinary NTx (Osteomark, Osteox International, Seattle, WA) was measured using an enzyme-linked immunosorbent assay. After overnight fasting, serum and first void urine samples were collected between 8:30 a.m. and 10:00 a.m. Immunoassays were performed by SRL, Inc. (Tokyo, Japan). Serum concentration 
of corrected calcium $(\mathrm{Ca})$ also was determined by conventional methods.

\section{Definition of responsive and non-responsive BP groups}

If $\mathrm{BMD}$ at the baseline minus that at 2 years earlier was negative, the subject was determined to be a non-responder to BP treatment. If the total was positive, the subject was defined as a $\mathrm{BP}$ responder. Since $5 \mathrm{BMD}$ readings were obtained over the 2 years prior to denosumab switching, the slopes of linear regression lines were calculated. A slope of $<0$ indicated a non-responder status.

Prior to final group classification, we compared the groups determined by 2 -point data (i.e., baseline and
2 years beforehand) with those determined by the slopes of linear regression lines using kappa statistics. As shown in Table 3, 68 as non-responders for the lumber spine and 30 as responders, while 67 as non-responders for the hip and 31 as responders. No significant associations between the groups were observed in this study for the lumbar spine or hips.

\section{Data analysis}

The independent $t$ test was used to investigate for differences in age, duration of BP use, concentrations of serum corrected $\mathrm{Ca}, \mathrm{BAP}$, and urinary NTx, BMD at 2 years prior to baseline, $\mathrm{BMD}$ at baseline, change in BMD from 2 years before
Fig. 1 a Change in BMD at lumbar spine. In both groups, lumbar BMD values increased significantly from before 2 years to after 1 year of denosumab treatment $(P<0.001)$. However, BMD values in the nonresponsive group did not reach those in the BP-responsive group at 1 year of denosumab therapy $(P=0.080)$. b Change in BMD at hips. In the non-responsive group, BMD values were significantly decreased $(P<0.001)$ after 2 years of BP treatment. After denosmab treatment, hip BMD values increased in both groups to a comparable degree $(P=0.709)$. As a result, BMD values in the non-responsive group were lower than those in the responsive group at 1 year of denosumab treatment $(P=0.065)$.

\section{a}

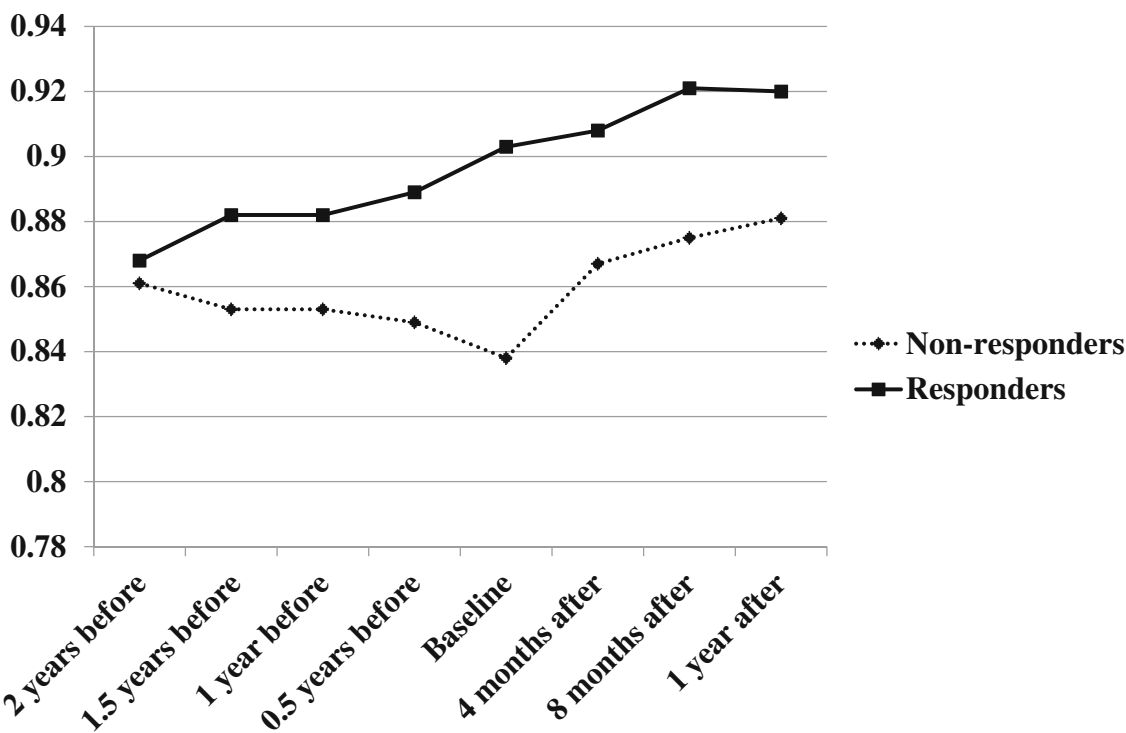

b

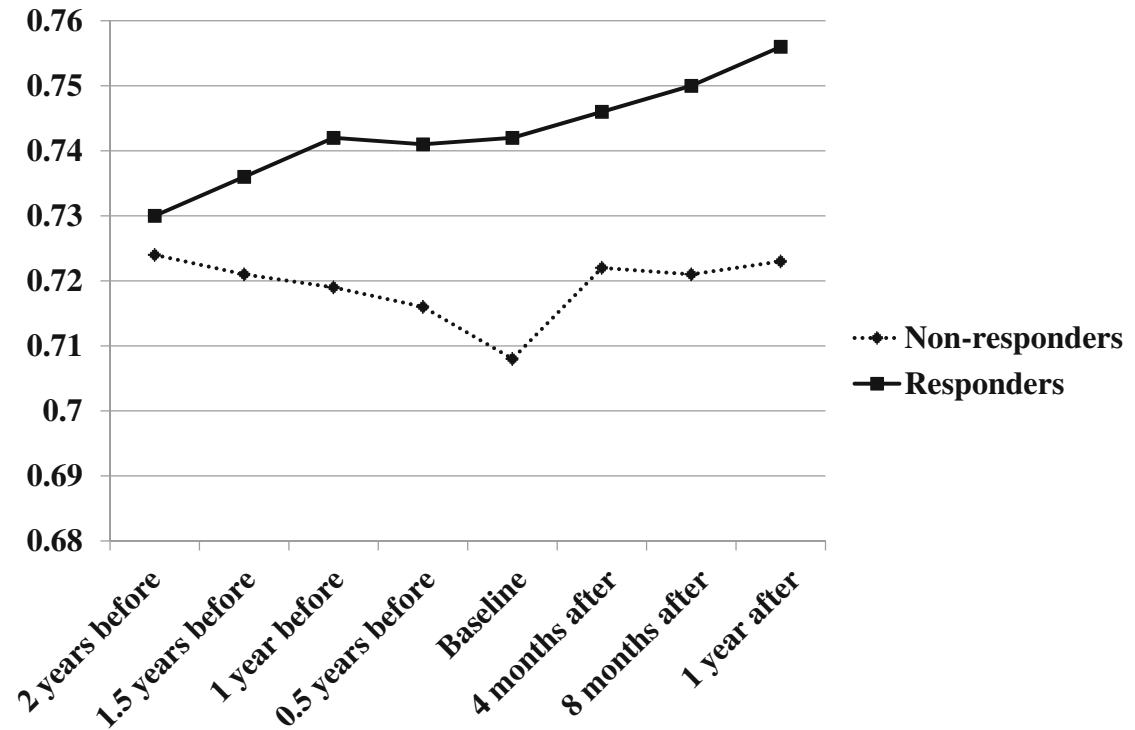


baseline, BMD after 1 year of denosumab treatment, and change in BMD from baseline to 1 year of denosumab between responders and non-responders at the lumbar spine and hips.

Comparisons between measurement points ( 2 years ago, baseline, and 1 year later) were done using repeated ANOVA with Bonferroni correction.

Simple correlation coefficients were calculated for BMD changes of the lumbar spine and hips for the 2 years of BP pretreatment and 1 year of denosumab therapy. Multiple linear regression analysis with the stepwise method adjusted for age, duration of BP treatment, and concentrations of serum corrected $\mathrm{Ca}$, BAP, and urinary NTx was used to evaluate for associations for the same periods. All data were analyzed using the Statistical Package for the Social Sciences (SPSS, version 19.0; IBM Inc., Chicago, IL, USA). $P$ values of less than 0.05 were considered statistically significant.

This investigation was performed in accordance with the ethical tenets set forth in the revised 2014 Declaration of Helsinki. The study was approved by the Institutional Ethics Committee at Showa Inan General Hospital, and informed written consent was obtained from all patients.

\section{Results}

The overall characteristics of the study subjects are summarized in Table 1 and overall results are presented in Fig. 1a, b. Bone turnover markers became significantly decreased after denosumab administration; mean (SD) BAP values before the treatment change were $10.9(3.7) \mu \mathrm{g} / \mathrm{L}$, while those at 4 months of denosumab were $7.8(2.5) \mu \mathrm{g} / \mathrm{L}(P<0.001)$. Mean (SD) urinary NTX values just before denosumab were $24.9(10.2) \mathrm{nmol} \mathrm{BCE} / \mathrm{mmol} \mathrm{Cr}$, and those at 4 months were $13.2(6.8) \mathrm{nmol} \mathrm{BCE} / \mathrm{mmol} \mathrm{Cr}(P<0.001)$.

At the lumbar spine, there were no significant differences in age, duration of $\mathrm{BP}$ use, serum $\mathrm{Ca}$, serum $\mathrm{BAP}$, urinary NTx, or BMD at baseline between the groups (Table 2). In the responsive group, BMD values were significantly increased $(P<0.001)$ after 2 years of BP treatment, which were significantly decreased $(P<0.001)$ in the non-responsive group. At this point, a significant difference was observed for lumbar BMD at the baseline $(P=0.021)$ between the test groups. After denosumab administration, BMD values increased in both groups. There was no significant difference in the increase of lumbar BMD values $(P=0.147)$, although the augmentation tended to be greater in the non-responsive group. As a result, the difference in BMD values between the groups decreased after a year of denosumab treatment to become comparable $(P=0.080)$. In both groups, lumbar BMD values increased significantly over the study period $(P<0.001)$. However, BMD readings in the non-responsive group remained lower compared with responsive group at 1 year of denosumab treatment (Fig. 1a).

At the hips, there were no significant differences in age, duration of BP use, serum Ca, serum BAP, urinary NTx, or $\mathrm{BMD}$ at 2 years prior to baseline between the groups (Table 3). Similarly to the lumbar data, in the responsive group, BMD values were significantly increased, while those for non-responders were significantly decreased, after 2 years of BP treatment. However, a significant difference was not observed for hip BMD at the baseline $(P=0.053)$. After denosumab initiation, hip BMD values increased comparably in both groups $(P=0.709)$, and thus, the BMD in the nonresponsive group remained lower than that in the responsive group $(P=0.065 ;$ Fig. $1 b)$. In the responsive group, hip BMD values increased significantly over the study period $(P<0.001)$. However, over the study period, hip BMD in the non-responsive group did not change or significantly increase.

Simple correlation coefficients were -0.337 for the lumbar spine and -0.339 for the hips (both $P=0.001$ ) for BMD changes over the study period (Fig. 2a, b). The less lumbar and hip BMD values were increased by initial BPs, the more significantly BMD values were increased by denosumab.

Table 1 Characteristics of 98 study subjects

\begin{tabular}{|c|c|}
\hline & Mean (SD) \\
\hline Age (years) & $71.2(6.9)$ \\
\hline Duration of BP use (months) & $59.9(34.3)$ \\
\hline Serum concentration of corrected calcium $(\mathrm{mg} / \mathrm{dl})$ & $9.20(0.40)$ \\
\hline Serum BAP $(\mu \mathrm{g} / \mathrm{L})$ & $10.9(3.7)$ \\
\hline Urinary NTx (nmol BCE/mmol Cr) & $24.9(10.2)$ \\
\hline $\begin{array}{l}\text { BMD of the lumbar spine } 2 \text { years before baseline } \\
\left(\mathrm{g} / \mathrm{cm}^{2}\right)\end{array}$ & $0.863(0.097)$ \\
\hline BMD of the lumbar spine at baseline $\left(\mathrm{g} / \mathrm{cm}^{2}\right)$ & $0.854(0.100)$ \\
\hline $\begin{array}{l}\text { Change of BMD from } 2 \text { years before baseline at } \\
\text { the lumbar spine }\left(\mathrm{g} / \mathrm{cm}^{2}\right)\end{array}$ & $-0.009(0.027)$ \\
\hline $\begin{array}{l}\text { BMD of the lumbar spine at } 1 \text { year of } \\
\text { denosumab treatment }\left(\mathrm{g} / \mathrm{cm}^{2}\right)\end{array}$ & $0.893(0.105)$ \\
\hline $\begin{array}{l}\text { Change of BMD from baseline to } 1 \text { year later at } \\
\text { the lumbar spine for denosumab treatment } \\
\left(\mathrm{g} / \mathrm{cm}^{2}\right)\end{array}$ & $0.040(0.031)$ \\
\hline $\begin{array}{l}\text { BMD of the hip } 2 \text { years before baseline } \\
\left(\mathrm{g} / \mathrm{cm}^{2}\right)\end{array}$ & $0.726(0.079)$ \\
\hline BMD of the hip at baseline $\left(\mathrm{g} / \mathrm{cm}^{2}\right)$ & $0.719(0.081)$ \\
\hline $\begin{array}{l}\text { Change of BMD from } 2 \text { years before baseline } \\
\text { at the hip at }\left(\mathrm{g} / \mathrm{cm}^{2}\right)\end{array}$ & $-0.007(0.020)$ \\
\hline $\begin{array}{l}\text { BMD of the hip } 1 \text { year of denosumab } \\
\text { treatment }\left(\mathrm{g} / \mathrm{cm}^{2}\right)\end{array}$ & $0.733(0.082)$ \\
\hline $\begin{array}{l}\text { Change of BMD from baseline to } 1 \text { year later } \\
\text { at hip for denosumab treatment }\left(\mathrm{g} / \mathrm{cm}^{2}\right)\end{array}$ & $0.015(0.015)$ \\
\hline
\end{tabular}

$B M D$ bone mineral density, $B A P$ bone specific alkaline phosphatase, $N T x$ $\mathrm{N}$-terminal telopeptide of type I collagen corrected by creatinine, $B P$ bisphosphonate 
Table 2 Differences in some parameters between responders and non-responders for BP pre-treatment at the lumbar spine

\begin{tabular}{|c|c|c|c|}
\hline & Non-responders & Responders & $P$ value \\
\hline Number of patients & 68 & 30 & \\
\hline Age (years) & $70.7(0.8)$ & $72.3(1.3)$ & 0.303 \\
\hline Duration of BP use (months) & $59.0(4.1)$ & $62.0(6.6)$ & 0.694 \\
\hline Serum corrected calcium (mg/dl) & $9.22(0.05)$ & $9.15(0.06)$ & 0.420 \\
\hline Serum BAP $(\mu \mathrm{g} / \mathrm{L})$ & $11.1(0.5)$ & $10.6(0.5)$ & 0.610 \\
\hline Urinary NTx (nmol BCE/mmol Cr) & $25.4(1.2)$ & $23.6(1.9)$ & 0.420 \\
\hline $\begin{array}{l}\text { BMD of the lumbar spine } 2 \text { years before } \\
\text { baseline }\left(\mathrm{g} / \mathrm{cm}^{2}\right)\end{array}$ & $0.861(0.011)$ & $0.868(0.019)$ & 0.736 \\
\hline BMD of the lumbar spine at baseline $\left(\mathrm{g} / \mathrm{cm}^{2}\right)$ & $0.838(0.011)$ & $0.889(0.019)$ & 0.021 \\
\hline $\begin{array}{l}\text { Change of BMD from } 2 \text { years before baseline } \\
\text { at the lumbar spine }\left(\mathrm{g} / \mathrm{cm}^{2}\right)\end{array}$ & $-0.022(0.002)$ & $0.021(0.003)$ & $<0.001$ \\
\hline $\begin{array}{l}\text { BMD of the lumbar spine at } 1 \text { year of } \\
\text { denosumab treatment }\left(\mathrm{g} / \mathrm{cm}^{2}\right)\end{array}$ & $0.881(0.011)$ & $0.921(0.022)$ & 0.080 \\
\hline $\begin{array}{l}\text { Change of BMD from baseline to } 1 \text { year later } \\
\text { at the lumbar spine for denosumab } \\
\text { treatment }\left(\mathrm{g} / \mathrm{cm}^{2}\right)\end{array}$ & $0.042(0.003)$ & $0.033(0.006)$ & 0.147 \\
\hline
\end{tabular}

Results were shown as mean (SEM)

$B M D$ bone mineral density, $B A P$ bone specific alkaline phosphatase, NTx N-terminal telopeptide of type I collagen corrected by creatinine, $B P$ bisphosphonate
Multiple linear regression analysis revealed that a decrease in BMD from 2 years before baseline to baseline $(t$ value $=-3.502, P=0.001)$ was significantly associated with an increased change in BMD by denosumab treatment at the lumbar spine (adjusted $R$ square $=0.113$ ). At the hips, multiple linear regression analysis also showed that a decrease in BMD from 2 years before baseline to baseline ( $t$ value $=-3.526 P=0.001$ ) was significantly associated with an increased change in BMD by denosumab therapy (adjusted $R$ square $=0.115$ ).

\section{Discussion}

BPs usually increase BMD in patients with OP, especially over the first few years, although the effectiveness of BPs on BMD diminishes over extended periods [7]. At our institutions, BPs given to osteoporotic patients with low BMD regularly increase BMD during initial treatment. However, some cases of plateauing, or even BMD decrease, are encountered regardless of the BP. This study showed that in non-responsive patients, many of whom
Table 3 Differences in some parameters between responders and non-responders for BP pretreatment at the hip

\begin{tabular}{|c|c|c|c|}
\hline & Non-responders & Responders & $P$ value \\
\hline Number of patients & 67 & 31 & \\
\hline Age (years) & $71.6(0.9)$ & $71.3(0.1)$ & 0.369 \\
\hline Duration of BP use (months) & $60.2(4.3)$ & $59.4(6.0)$ & 0.916 \\
\hline Serum concentration of corrected calcium (mg/dl) & $9.20(0.05)$ & $9.18(0.06)$ & 0.840 \\
\hline Serum BAP $(\mu \mathrm{g} / \mathrm{L})$ & $11.0(0.5)$ & $10.7(0.6)$ & 0.731 \\
\hline Urinary NTx (nmol BCE/mmol Cr) & $24.3(1.1)$ & $26.0(2.3)$ & 0.461 \\
\hline $\begin{array}{l}\text { BMD of the hip } 2 \text { years before basseline } \\
\left(\mathrm{g} / \mathrm{cm}^{2}\right)\end{array}$ & $0.724(0.010)$ & $0.730(0.011)$ & 0.732 \\
\hline BMD of the hip at baseline $\left(\mathrm{g} / \mathrm{cm}^{2}\right)$ & $0.708(0.010)$ & $0.742(0.011)$ & 0.053 \\
\hline $\begin{array}{l}\text { Change of BMD from } 2 \text { years before baseline at } \\
\text { the hip at }\left(\mathrm{g} / \mathrm{cm}^{2}\right)\end{array}$ & $-0.015(0.002)$ & $0.012(0.002)$ & $<0.001$ \\
\hline $\begin{array}{l}\text { BMD of the hip } 1 \text { year of denosumab } \\
\text { treatment }\left(\mathrm{g} / \mathrm{cm}^{2}\right)\end{array}$ & $0.723(0.010)$ & $0.756(0.011)$ & 0.065 \\
\hline $\begin{array}{l}\text { Change of BMD from baseline to } 1 \text { year later at } \\
\text { the hip for denosumab treatment }\left(\mathrm{g} / \mathrm{cm}^{2}\right)\end{array}$ & $0.015(0.002)$ & $0.014(0.002)$ & 0.709 \\
\hline
\end{tabular}

Results were shown as mean (SEM)

$B M D$ bone mineral density, $B A P$ bone specific alkaline phosphatase, $N T x$ N-terminal telopeptide of type I collagen corrected by creatinine, $B P$ bisphosphonate 
Fig.2 a Simple correlation coefficient for lumbar spine. Simple correlation coefficient was -0.337 for BMD at the lumbar spine $(P=0.001)$ for 2 years of BP treatment and 1 year of denosumab treatment. The lower was the effect on BMD by BPs, the more significant was the effect of denosumab treatment on BMD amelioration. b Simple correlation coefficient for hips. Simple correlation coefficient was -0.339 for BMD at the hip $(P=0.001)$ for 2 years of BP treatment and 1 year of denosumab treatment. The lower was the effect on BMD by BPs, the more significant was the effect of denosumab treatment on BMD amelioration. a

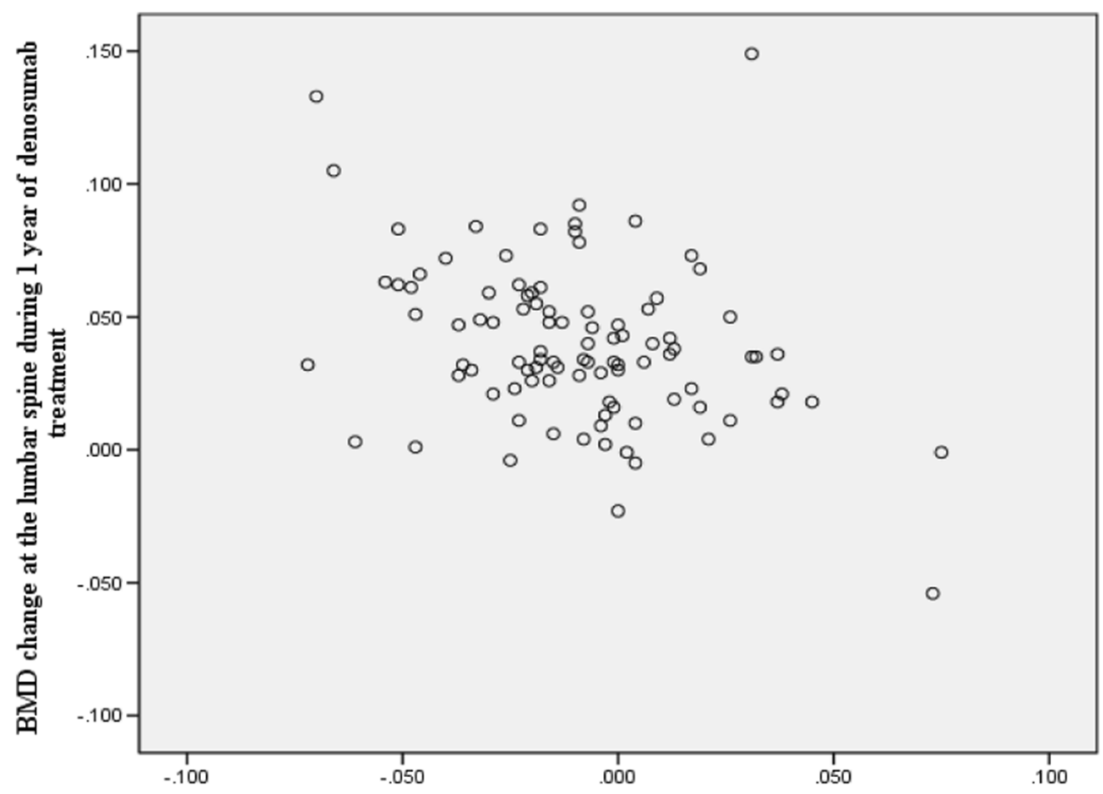

$\mathrm{BMD}$ change at the lumbar spine during last 2 years of $\mathrm{BP}$ pre-treatment

b

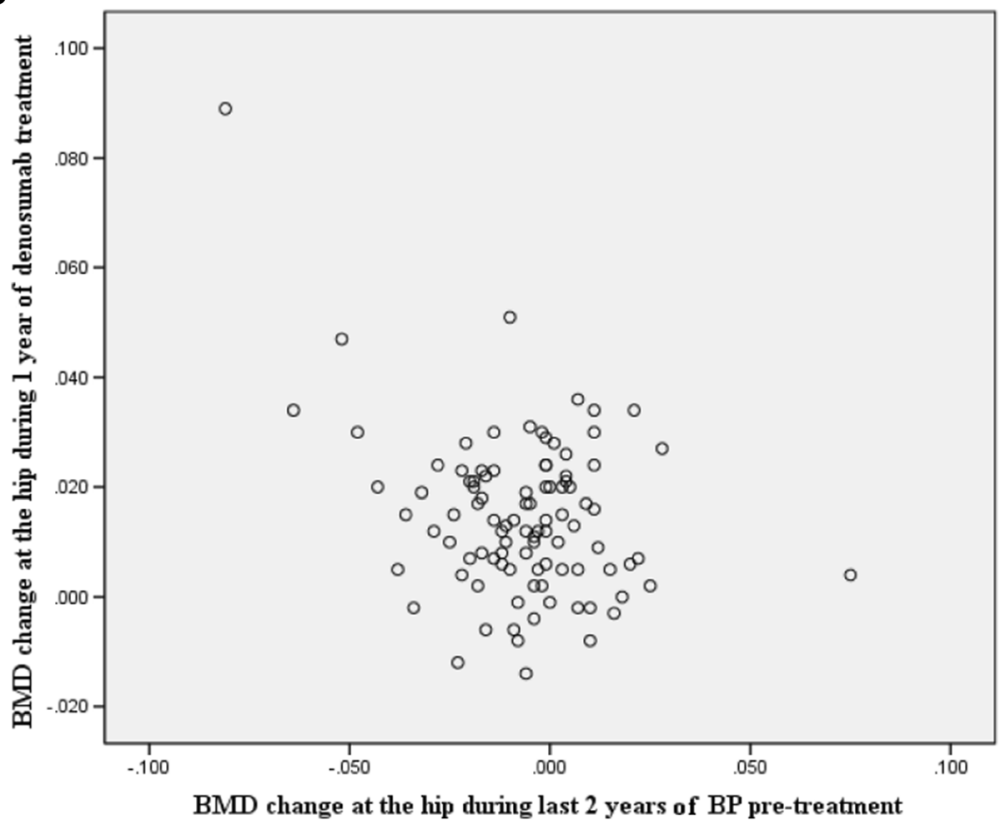

had been taking BPs for over 5 years, both lumbar and hip BMD became significantly decreased over time. These findings confirmed that BP non-responsiveness developed during long-term BP treatment in patients with OP.

Recent literature on denosumab has shown that the drug produces greater increases in BMD than do BPs as well as a persistent, almost linear increase in spine, total hip, and femoral neck BMD beyond 3 years and up to 8 years [10]. It is believed that denosumab differs from $\mathrm{BPs}$, for which the rate of BMD improvement diminishes and for some drugs becomes negative 2-3 years later, when the process of secondary mineralization flattens out [10]. However, the mechanism of reduced BMD after prolonged BPs remains unknown.

In this study on the switch from BPs to denosumab, the bone turnover markers that had been inhibited by BPs further decreased significantly by denosumab. According to direct comparative studies, denosumab increases significantly greater BMD values than do BPs [11]. There have been several reports describing the effects of denosumab after BP treatment. Roux et al. found that even after BP treatment, denosumab had stronger inhibitory effects on bone resorption 
and stimulatory effects on BMD [12], confirming that denosumab produced much more pronounced inhibition of bone resorption.

Denosumab is well recognized as a potent antiresorptive agent. However, there have been no reports on the effects of denosumab in BP-unresponsive cases. Our results showed that a switch to denosumab from BPs significantly increased BMD values that had even become decreased by BP therapy, and thus, was effective in non-responsive patients. Furthermore, this study revealed that a decrease in BMD from 2 years prior to baseline to baseline using BP treatment was significantly associated with an enhanced BMD change by denosumab both at the lumbar spine and hips. The mechanism of the increase in BMD caused by denosumab in the non-responsive group, apart from stronger inhibitory effects on bone resorption, is unclear. We previously compared the values of bone turnover markers treated by denosumab without BP pre-treatment with those after long-term BP treatment. In the pre-treated group, bone resorption markers were significantly inhibited after only 1 week of denosumab therapy, while bone formation markers did not change remarkably, even at 4 months. We speculate that such bone metabolic inhibitory differences may indicate a BMD increase [13].

There have been several reports on the effects of PTH after BP treatment [14]. Blumsohn et al. stated that BMD values increased by PTH in BP-unresponsive cases. However, in our experience, $\mathrm{PTH}$ after BP treatment is a secondary option since the impact of PTH after BPs is markedly lower in Japanese patients [15]. In contrast, denosumab produced significant BMD improvements after BP therapy, and may therefore represent a better treatment than PTH in Japanese BPunresponsive cases.

Our findings showed that BMD values decreased substantially in non-responsive cases with time and a switch to denosumab increased BMD values. Furthermore, this study revealed that decreased BMD by prolonged BP pretreatment was significantly associated with increased BMD after denosumab administration both in the lumbar spine and hips. These results suggesting that patients exhibiting diminished BP therapy response should immediately change to denosumab.

The main limitations of this study are its retrospective nature and that a long-term observational period will be needed to clarify whether or not BMD will increase continuously by denosumab and to what extent fractures will be prevented.

In this study, clinical treatment results were analyzed retrospectively. Thus, there were many subjects who received extremely long-term BP treatment that could be observed for more than 2 years for BP responsiveness. Moreover, individual BP treatments varied since the drugs were changed to denosumab in the case of a poor BP response after denosumab approval in Japan in 2013. Based on our results, treatment should be changed as soon as possible if a BMD increase is not achieved. Further prospective studies will be challenging as it may be impossible to perform similar studies to ours from now on from the ethical standpoint.

In summary, BMD values increased by denosumab following BP treatment, even in BP-unresponsive osteoporotic cases. Thus, it might not be appropriate to continue BPs in such patients, who should urgently be switched to denosumab.

\section{Compliance with ethical standards}

\section{Conflicts of interest None}

Open Access This article is distributed under the terms of the Creative Commons Attribution-NonCommercial 4.0 International License (http:// creativecommons.org/licenses/by-nc/4.0/), which permits any noncommercial use, distribution, and reproduction in any medium, provided you give appropriate credit to the original author(s) and the source, provide a link to the Creative Commons license, and indicate if changes were made.

\section{References}

1. McClung MR, Lewiecki EM, Cohen SB, Bolognese MA, Woodson GC, Moffett AH, Peacock M, Miller PD, Lederman SN, Chesnut CH, Lain D, Kivitz AJ, Holloway DL, Zhang C, Peterson MC, Bekker PJ (2006) AMG 162 bone loss study group. Denosumab in postmenopausal women with low bone mineral density. N Engl J Med 354:821-831

2. Silva I, Branco JC (2012) Denosumab: recent update in postmenopausal osteoporosis. Acta Reumatol Port 37:302-313 Review

3. Ferrari S, Adachi JD, Lippuner K, Zapalowski C, Miller PD, Reginster JY, Törring O, Kendler DL, Daizadeh NS, Wang A, O'Malley CD, Wagman RB, Libanati C, Lewiecki EM (2015) Further reductions in nonvertebral fracture rate with long-term denosumab treatment in the FREEDOM open-label extension and influence of hip bone mineral density after 3 years. Osteoporos Int 26:2763-2771

4. Iwamoto J, Sato Y, Takeda T, Matsumoto H (2008) Hip fracture protection by alendronate treatment in postmenopausal women with osteoporosis: a review of the literature. Clin Interv Aging 3: 483-489 Review

5. Stresing V, Fournier PG, Bellahcène A, Benzaïd I, Mönkkönen H, Colombel M, Ebetino FH, Castronovo V, Clézardin P (2011) Nitrogen-containing bisphosphonates can inhibit angiogenesis in vivo without the involvement of farnesyl pyrophosphate synthase. Bone 48:259-266

6. Russell RG (2011) Bisphosphonates: the first 40 years. Bone 49:219

7. Bone HG, Hosking D, Devogelaer JP, Tucci JR, Emkey RD, Tonino RP, Rodriguez-Portales JA, Downs RW, Gupta J, Santora AC, Liberman UA (2004) Alendronate phase III osteoporosis treatment study group. Ten years' experience with alendronate for osteoporosis in postmenopausal women. N Engl J Med 350:1189-1199

8. Reyes C, Hitz M, Prieto-Alhambra D, Abrahamsen B (2016) Risks and benefits of bisphosphonate therapies. J Cell Biochem 117:20-28

9. Ikegami S, Kamimura M, Uchiyama S, Nakamura Y, Mukaiyama K, Kato H (2016) Clinical implications of hip flexion in the measurement of spinal bone mineral density. J Clin Densitom 19:270-276 
10. Törring O (2015) Effects of denosumab on bone density, mass and strength in women with postmenopausal osteoporosis. Ther Adv Musculoskelet Dis 7:88-102

11. Brown JP, Prince RL, Deal C, Recker RR, Kiel DP, de Gregorio LH, Hadji P, Hofbauer LC, Alvaro-Gracia JM, Wang H, Austin M, Wagman RB, Newmark R, Libanati C, San Martin J, Bone HG (2009) Comparison of the effect of denosumab and alendronate on BMD and biochemical markers of bone turnover in postmenopausal women with low bone mass: a randomized, blinded, phase 3 trial. J Bone Miner Res 24:153-161

12. Roux C, Hofbauer LC, Ho PR, Wark JD, Zillikens MC, FahrleitnerPammer A, Hawkins F, Micaelo M, Minisola S, Papaioannou N, Stone M, Ferreira I, Siddhanti S, Wagman RB, Brown JP (2014) Denosumab compared with risedronate in postmenopausal women suboptimally adherent to alendronate therapy: efficacy and safety results from a randomized open-label study. Bone 58:48-54
13. Nakamura Y, Kamimura M, Ikegami S, Mukaiyama K, Uchiyama S, Taguchi A, Kato H (2015) Changes in serum vitamin D and PTH values using denosumab with or without bisphosphonate pretreatment in osteoporotic patients: a short-term study. BMC Endocr Disord 15:81

14. Kamimura M, Nakamura Y, Ikegami S, Iinou N, Mukaiyama K, Uchiyama S, Kato H (2016) Bisphosphonate pretreatment diminishes the therapeutic benefits of teriparatide in Japanese osteoporotic patients. Tohoku J Exp Med 239: $17-24$

15. Blumsohn A, Marin F, Nickelsen T, Brixen K, Sigurdsson G, González de la Vera J, Boonen S, Liu-Léage S, Barker C, Eastell R, EUROFORS Study Group (2011) Early changes in biochemical markers of bone turnover and their relationship with bone mineral density changes after 24 months of treatment with teriparatide. Osteoporos Int 22:1935-1946 